submitted to ApJLetters

\title{
Some constraints on the lower mass limit for double-degenerate progenitors of Type Ia supernovae
}

\author{
X. Chen ${ }^{1,2}$, C. S. Jeffery ${ }^{3,4}$, X. Zhang ${ }^{3}$ and Z. $\operatorname{Han}^{1,2}$, \\ ${ }^{1}$ National Astronomical Observatories / Yunnan Observatory, Chinese Academy of \\ Sciences, Kunming, 650011, China \\ ${ }^{2}$ Key Laboratory for the Structure and Evolution of Celestial Objects, Chinese Academy of \\ Sciences, Kunming, 650011, China \\ ${ }^{3}$ Armagh Observatory, College Hill, Armagh BT61 9DG \\ ${ }^{4}$ School of Physics, Trinity College Dublin, Dublin 2, Ireland \\ xuefeichen717@hotmail.com
}

\begin{abstract}
Recent theoretical and observational studies both argue that the merging of double carbon-oxygen white dwarfs (WDs) is responsible for at least some Type Ia supernovae (SNe Ia). Previous (standard) studies of the anticipated SN birthrate from this channel have assumed that the merger process is conservative and that the primary criterion for explosion is that the merged mass exceeds the Chandrasekhar mass. Han \& Webbink (1999) demonstrated that mass transfer and merger in close double WDs will in many cases be non-conservative. Pakmor et al. (2011) further suggested that the merger process should be violent in order to initiate an explosion. We have therefore investigated how the SN Ia birthrate from the double-degenerate (DD) channel is affected by these constraints. Using the binary-star population-synthesis method, we have calculated the DD SN Ia birthrate under conservative and non-conservative approximations, and including lower mass and mass-ratio limits indicated by recent smoothedparticle-hydrodynamic calculations. The predicted DD SN Ia rate is significantly reduced by all of these constraints. With dynamical mass loss alone (violent merger) the birthrate is reduced to $56 \%$ of the conservative rate. Requiring that the mass ratio $q>2 / 3$ further reduces the birthrate to $18 \%$ that of the standard assumption. An upper limit of $0.0061 \mathrm{SNuM}$, or a Galactic rate of $4.6 \times 10^{-4} \mathrm{yr}^{-1}$, might be realistic.
\end{abstract}

Subject headings: binaries: close — stars: evolution — supernovae: general 


\section{Introduction}

The double-degenerate (DD) merger was suggested as a possible channel for type Ia supernovae (SNe Ia) in the early 1980's (Tutukov \& Yungelson 1981; Iben \& Tutukov 1984; Webbink 1984). In this DD model, two carbon-oxygen (CO) white dwarfs (WDs) can produce a SN Ia while merging if their total mass is larger than the Chandrasekhar mass $\left(M_{\mathrm{ch}}\right)$. The DD model can naturally explain the lack of $\mathrm{H}$ and He emission in the spectra of SNe Ia and some super-luminous SNe Ia, but it has a major difficulty in explaining the similarities of most SNe Ia since the merger mass has a relatively wide range, $\sim 1.4-2.0 M_{\odot}$ (Wang et al. 2010)1. Meanwhile, although the SNe Ia rate predicted from the DD model is comparable to that of observations (Yungelson et al. 1994; Han 1998; Nelemans et al. 2001; Ruiter, Belczynski \& Fryer 2009; Wang et al. 2010; Yu \& Jeffery 2010), theoretical studies suggest that the merger of two WDs is more likely to lead to an accretioninduced collapse (AIC) to form a neutron star (Nomoto \& Iben 1985; Saio \& Nomoto 1985; Timmes, Woosley \& Taam 1994). Consequently, the DD model has been disadvantaged in comparison to the single-degenerate (SD) model for a long time (Wang \& Han 2012).

Piersanti et al. (2003) suggested that, under the right conditions, the DD merger process could be quite violent and lead to a SN Ia explosion rather than AIC (see also Yoon, Podsiadlowski \& Rosswog (2007); Shen et al. (2012)). Fully three-dimensional simulations of a violent merger of two CO WDs by Pakmor et al. (2010, 2011) show that the merger of two equal-mass CO WDs $\left(\sim 0.9 M_{\odot}\right)$ can explain the formation of sub-luminous 1991bg-like events (see also van Kerkwijk, Chang \& Justham (2010)). The Pakmor et al. (2012) simulation of a DD merger with masses of $1.1 M_{\odot}$ and $0.9 M_{\odot}$ shows good agreement with properties of normal SNe Ia. Observationally, some known or potential DD systems such as WD 2020-425 (Napiwotzki et al. 2007), V458 Vulpeculae (Rodríguez-Gil et al. 2010), SBS1150+599A (Tovmassian et al. 2010) and GD687 (Geier et al. 2010) represent good candidates for SN Ia progenitors since they have total masses close to $M_{\mathrm{ch}}$ and orbital periods short enough to merge within a Hubble time. Furthermore, extensive searches have found no surviving companion to the Type Ia supernovae responsible for SNR 0509-67.5 (Schaefer \& Pagnotta 2012) or SN 1572 (Kerzendorf et al. 2012), whence it is argued that the progenitors must have been DD systems. Hence, it appears that at least some, if not most, SNe Ia come from DD mergers.

Previous studies for the DD model generally assume that a SN Ia is produced if the

\footnotetext{
${ }^{1}$ It can be argued, however, that in the DD scenario it is the primary (exploding) WD which matters most, not the total mass, since it is the detonation in the exploding WD which is primarily responsible for synthesizing ${ }^{56} \mathrm{Ni}$ (Pakmor et al. 2010, 2012).
} 
total mass of a DD system is larger than $M_{\mathrm{ch}}$. This assumption implies that the DD merger process is always conservative. However, if accretion is spherically symmetric, mass transfer can be dramatically non-conservative both during stable mass transfer and in a violent merger. Han \& Webbink (1999) (HW) studied the stability and energetics of mass transfer in double WDs. They showed that the expelled mass fraction of an interacting double WD depends on the component masses and can be as large as $50 \%$ of the donor for a system with masses of $1 M_{\odot}$ and $0.5 M_{\odot}$. Meanwhile, smoothed-particle-hydrodynamic (SPH) simulations (Yoon, Podsiadlowski \& Rosswog 2007; Pakmor et al. 2011) have shown that not all double CO WDs with a total mass larger than $M_{\text {ch }}$ can produce SNe Ia. For example, Pakmor et al. (2011) find that only double CO WDs in which the more massive white dwarf exceeds $\sim 0.9 M_{\odot}$ and the mass ratio roughly exceeds 0.8 robustly reach the conditions required for initiating a detonation, leading to a SN Ia explosion. Obviously, some additional constraints should be placed on the DD model for triggering SNe Ia 2 .

In sect.2 we investigate the merger mass of double WDs including non-conservative mass transfer, and we examine the impact of these constraints and those arising from SPH simulations on the SNe Ia birthrate in sect.3. Conclusions are drawn in sect.4.

\section{The Merger Mass}

As the orbit of a double WD system decays due to the emission of gravitational radiation/waves, the less massive component will be the first to fill its Roche lobe and become the mass donor; the more massive component becomes the accretor. Unlike non-degenerate donor stars in other compact binaries, the WD donors in double WDs lie deeper within the potential well of the accreting star, resulting in important consequences for mass transfer. Assuming energy conservation and hence no energy sources other than gravitational potential energy are at play, HW analyzed the initial stability of this mass transfer and the fraction of the mass-transfer stream to be expelled from the binary. From this point of view, the mass transfer is conservative if the accretion luminosity is less than the Eddington limit. Any accretion luminosity in excess of the Eddington limit is assumed to be absorbed in the accretion flow. In terms of energetics, radiative losses represent accretion energy not used to power a mass outflow (HW: Eq (21)). The fraction of matter accreted by the primary is used to power the outflow and can be determined from HW: Eq (22). For component masses

\footnotetext{
${ }^{2}$ From the point of view of preventing an off-center C ignition, Yoon, Podsiadlowski \& Rosswog (2007) favored less massive double CO WDs to produce SNe Ia. Since an off-center C ignition is almost inevitable (Shen et al. 2012), and much of the physics for the thermal evolution of merger remnants is unclear, we only consider here the constraints from Pakmor et al. (2011).
} 
$M_{1}$ (accretor) and $M_{2}$ (donor), we adopt HW: Eqs (17), (22) and (23) to calculate the mass transfer rate, $\dot{M}_{2}$, and the fraction of matter to be accreted by the primary 3 , $\beta$.

There are two regimes for computing the final total mass of the merger, $M$. If the merger process is dynamical, $\beta$ can be applied as a single step process and $M=M_{1}+\beta M_{2}$. If mass transfer is stable, $M$ is obtained by integrating over the mass-transfer epoch 4 , using $M=M_{1}+\int \beta(t) \dot{M}_{2}(t) \mathrm{d} t$.

\section{Type Ia supernovae from the DD model}

\subsection{The formation of double CO WD systems}

To investigate the SN Ia birthrate due to DD mergers, we require a population of close double CO WD binaries, to be formed from interactions in a population of primordial binary stars (e.g. Han (1998)). We first perform a Monte-Carlo simulation to obtain a stellar population, then evolve them in a rapid binary evolution code (RBEC) (Hurley, Tout \& Pols 2000, 2002) to obtain a sample of close CO WD systems.

In the Monte Carlo simulation, all stars are assumed to be members of binaries and have circular orbits. The primaries follow the initial mass function of Miller \& Scalo (1979) and are generated according to the formula of Eggleton. Tout \& Fitechett (1989), in the mass range 0.08 to $100 M_{\odot}$. The secondary mass, also with a lower limit of $0.08 M_{\odot}$, is then obtained from a constant mass-ratio distribution. The distribution of orbital separations $a$ is taken to be constant in $\log a$ for wide binaries. This separation distribution has been used in many Monte-Carlo simulations and implies an equal number of wide binary systems per logarithmic interval and approximately 50 per cent of stellar systems with orbital periods less than 100 yr (Han 1998). Long-orbital-period binaries are effectively single stars.

The formation of close double CO WD systems depends significantly on the critical mass ratio for dynamical instability $q_{\mathrm{c}}$ and common envelope (CE) evolution (Han 1998). A binary experiences stable Roche lobe overflow (RLOF) when $q \leq q_{\mathrm{c}}$ and CE evolution otherwise, where $q=$ donor/accretor. In this study we adopt equation (57) of Hurley, Tout \& Pols (2002) for $q_{\mathrm{c}}$ when the mass donor is on the first or asymptotic giant branch (AGB), and

\footnotetext{
${ }^{3}$ Note that the model of HW takes the accretion to be spherical. If mass transfer is through a disk, then excess energy can be radiated away from the poles and $\beta$ may not be appropriate.

${ }^{4}$ The fitting formulae and a table for the merger mass from the two regimes for various component masses may be obtained by email from xuefeichen717@hotmail.com.
} 
let $q_{\mathrm{c}}=4$ when the mass donor is on the main sequence or in the Hertzsprung gap, as supported by detailed binary evolution studies (Han, Tout \& Eggleton 2000; Chen \& Han 2002, 2003). For CE evolution, we use the standard energy formalism (van den Heuvel 1976; Webbink 1984; Livio \& Soker 1988), that is, the CE is ejected if $\alpha_{\text {ce }} \Delta E_{\text {orb }} \geq E_{\text {bind }}$, where $\Delta E_{\text {orb }}$ is the orbital energy released, $\alpha_{\text {ce }}$ is the CE ejection efficiency, and $E_{\text {bind }}$ is the binding energy of the envelope and can be written as $G M_{\mathrm{d}} M_{\text {env }} /\left(\lambda R_{\mathrm{d}}\right)$ (where $M_{\mathrm{d}}, M_{\text {env }}$ and $R_{\mathrm{d}}$ are the mass, envelope mass and radius of the donor, respectively, and $\lambda$ is a structure parameter that depends on the evolutionary stage of the mass donor). We combine $\alpha_{\text {ce }}$ and $\lambda$ into one free parameter, setting $\alpha_{\mathrm{ce}} \lambda=1.5$ to reproduce the number of the DD objects in the Galaxy as in previous studies (Wang et al. 2009b).

RBEC distinguishes three types of WDs, namely helium (expected only in binaries), CO and oxygen-neon (ONe), respectively. The critical condition for $\mathrm{CO}$ and ONe WDs is the core mass at the base of AGB, $M_{\mathrm{c}, \mathrm{BAGB}}$. CO WDs are produced when $M_{\mathrm{c}, \mathrm{BAGB}}<1.6 M_{\odot}$, and $\mathrm{ONe}$ WDs when $1.6 M_{\odot} \leq M_{\mathrm{c}, \mathrm{BAGB}} \leq 2.5 M_{\odot}$, where $M_{\mathrm{c}, \mathrm{BAGB}}$ is given in $\mathrm{Eq}(66)$ of Pols et al. (1998). The mass of the CO core, and the final mass of CO WDs is determined by the $L-M_{\mathrm{c}}$ relation, i.e. $\operatorname{Eqs}(37)$ and (39) of that paper.

\subsection{Constraints on the progenitors of SNe Ia}

There are two basic constraints on double CO WDs as the progenitors of SNe Ia in the conservative case: (i) $M_{1}+M_{2}>M_{\mathrm{ch}} 5$, and (ii) the CO WDs are close enough to merge in a Hubble time. The timescale for two components to merge by gravitational wave radiation, $t_{\mathrm{GW}}$, is written as (Landau \& Lifshitz 1971)

$$
t_{\mathrm{GW}}=8 \times 10^{7} \times \frac{\left(M_{1}+M_{2}\right)^{1 / 3}}{M_{1} M_{2}} P^{8 / 3},
$$

where $P$ is the orbital period in hours, $t_{\mathrm{GW}}$ in years and $M_{1}, M_{2}$ in solar mass.

In the non-conservative case, (i) should be redefined as the final mass $M>M_{\mathrm{ch}}$ (see sect. 2). Furthermore, AIC should be avoided in order to successfully trigger a SN Ia. We therefore need additional constraints from dynamical simulations. Pakmor et al. (2010, 2011, 2012) showed that an initial detonation at the onset of $\mathrm{C}$ ignition can lead the DD merger to avoid AIC and explode, and that DDs with $M_{1} \geq 0.9 M_{\odot}$ and a mass ratio $M_{1} / M_{2} \gtrsim 0.8$ can achieve conditions for initiating such a detonation. Their studies also

\footnotetext{
${ }^{5}$ We adopt $M_{\mathrm{ch}}=1.378 M_{\odot}$ following Han \& Podsiadlowski (2004); Meng et al. (2009); Wang et al. (2009a).
} 
showed that mergers with $M_{1} \simeq 0.9 M_{\odot}$ are very promising candidates for explaining $s u b$ luminous SNe Ia, while a normal SN Ia can be produced from the double CO WDs with $M_{1}=1.1 M_{\odot}$. Since the SN Ia luminosity is determined by the amount of ${ }^{56} \mathrm{Ni}$ synthesized in the explosion, these simulation results seem to indicate that the primary mass is related to the final production of ${ }^{56} \mathrm{Ni}$, and therefore determines whether a normal or sub-luminous SN Ia is produced. Meanwhile, we do see a lot of variation in SN Ia lightcurves, and thus one might expect to see a variety among exploding WD masses provided by the DD models. In the absence of other constraints, we study the SN Ia birthrate assuming two lower mass limits for the DD accretor, i.e. $M_{1}>0.9$ or $1.0 M_{\odot}$, respectively.

Another constraint from the $\mathrm{SPH}$ simulations is the mass ratio of the CO DDs, $q=$ $M_{2} / M_{1}$. It has a critical value, $\approx 0.8$, below which the merger is not violent enough to ignite a detonation (Pakmor et al. 2011)6. The relation between the violence of a merger and the mass ratio can be simply understood from Fig. 2 of HW. In the dynamical instability region, $\zeta_{\mathrm{ad}}-\zeta_{\mathrm{L}}<0$, where $\zeta_{\mathrm{ad}}$ and $\zeta_{\mathrm{L}}$ are the adiabatic mass-radius exponent of the donor and the mass-radius exponent of its Roche radius, respectively. With the mass ratio closer to unity, the value of $\left|\zeta_{\mathrm{ad}}-\zeta_{\mathrm{L}}\right|$ becomes larger and larger. Since it is a negative number, the process of self-amplifying $\dot{M}_{2}$ (Eq (1) in that paper) is thus faster and faster, resulting in a more and more violent merger process. We arbitrarily relax the critical mass ratio from 0.8 to $2 / 3$ in our study to give an upper limit for the SN Ia birthrate from the SPH simulations.

In brief, based on the sample of double CO WDs which can merge in a Hubble time, we compute the SN Ia birthrate for the following cases :

(i) $M=M_{1}+\beta M_{2} \geq M_{\mathrm{ch}}$;

(ii) $M=M_{1}+\int \beta(t) \dot{M}_{2}(t) \mathrm{d} t \geq M_{\mathrm{ch}}$;

(iii) As case (ii) with $M_{1} \geq 0.9 M_{\odot}$. We choose case (ii) as the basic constraint since it includes a larger parameter space than case(i) (see Fig.1). The overall SNe Ia rate from case (ii) can then be considered as an upper limit from the non-conservative assumption.

(iv) similar to (iii) but $M_{1} \geq 1.0 M_{\odot}$, and

(v) an additional constraint, $q=M_{2} / M_{1} \geq 2 / 3$, is placed on case (iii).

Figure 1 shows these constraints in the $M_{1}-M_{2}$ plane (left panel), as well as CO DDs with a total mass $M_{1}+M_{2}>M_{\text {ch }}$ which can merge in a Hubble time (right panel). To obtain these DDs, we generate a population of $10^{7}$ binaries (with a total mass of $\simeq 1.26 \times 10^{7} M_{\odot}$ )

\footnotetext{
${ }^{6}$ Pakmor et al. (2011) found that the critical value of $q$ probably changes with primary WD mass; i.e. more massive primaries can merge with lower mass ratios.
} 
as described in sect.3.1 and evolve them in RBEC. These DDs cover a wide range in the $M_{1}-M_{2}$ plane, and also a large range of $\beta$. For $q=2 / 3$, the $\beta$ decreases from 0.7 to 0.54 as $M_{1}$ increases from 0.827 to $1.2 M_{\odot}$. The overall parameter spaces of SN Ia progenitors in cases (i)-(iv), i.e. to the right of the red lines, are reduced by different degrees relative to the conservative assumption, indicating corresponding decreases of the DD SN Ia rate under the non-conservative assumption and with dynamical constraints.

\subsection{Birth rates of $\mathrm{SNe} \mathrm{Ia}$}

The CO WDs above are used to study the SN Ia birthrates in various cases, which are finally normalized to (a) a single starburst of $10^{11} M_{\odot}$ and (b) a constant star formation rate of $5 M_{\odot} \mathrm{yr}^{-1}$ over the past $15 \mathrm{Gyr}$, to resemble our Galaxy. The results are shown in Fig.2. We see that, in the conservative case, the predicted SN Ia birthrate is $0.033 \mathrm{SNuM} 7$ (corresponding to a Galactic birth rate of $2.5 \times 10^{-3} \mathrm{yr}^{-1}$ ) for the constant star-formation at $15 \mathrm{Gyr}$ (left panel). In the non-conservative case, the predicted overall rate is reduced to $0.019 \mathrm{SNuM}\left(1.4 \times 10^{-3} \mathrm{yr}^{-1}\right)$ and $0.024 \mathrm{SNuM}\left(1.8 \times 10^{-3} \mathrm{yr}^{-1}\right)$ for cases (i) and (ii), respectively. If we introduce additional restrictions from dynamical simulations, the overall $\mathrm{SN}$ Ia rate is further reduced to $0.017 \mathrm{SNuM}\left(1.3 \times 10^{-3} \mathrm{yr}^{-1}\right), 0.0099 \mathrm{SNuM}\left(7.4 \times 10^{-4} \mathrm{yr}^{-1}\right)$ and $0.0061 \mathrm{SNuM}\left(4.6 \times 10^{-4} \mathrm{yr}^{-1}\right)$ for cases (iii)-(v), respectively. The birthrate in case (v) is only about $18 \%$ of that of the conservative case. Note that the SN Ia birthrate from case (v) might/could represent an upper limit based on constraints from recent SPH simulations (see sect.3.2).

Cases (i)-(iv) present similar delay-time distributions to that of the conservative case, that is, a peak around $0.8-3.2 \times 10^{8} \mathrm{yr}$ with a tail decaying as $t^{-1}$, where $t$ is the delay time (Fig. 2, right). The shape of this distribution is consistent with both theoretical analyses and observations (see Maoz et al. 2011; Wang \& Han 2012 and references therein). However, in case $(\mathrm{v})$, one sees a small peak around $\log t(\mathrm{yr})=8.7$ and a 'dip' just before $\log t(\mathrm{yr})=8.5$. RBEC produces CO WDs nearly uniformly distributed in orbital period. The $\mathrm{CE}+\mathrm{CE}$ channel produces extremely short orbital periods and the RLOF $+\mathrm{CE}$ channel gives relatively longer orbital periods, with some overlap between. Requiring $q \geq 2 / 3$ removes low- $M_{2}$ binaries, mainly from the RLOF $+\mathrm{CE}$ channel. These low $-M_{2}$ binaries are produced in two ways; i) the AGB progenitors of $M_{2}$ before the CE phase have low masses and generally lower binding energies and produce double CO WDs with longer orbital periods for a given $M_{1}$, ii) binaries with short orbital periods before CE ejection produce double CO WDs with short

\footnotetext{
${ }^{7} 1 \mathrm{SNuM}=1 \mathrm{SN}(100 \mathrm{yr})^{-1}\left(10^{10} \mathrm{M}_{\odot}\right)^{-1}$
} 

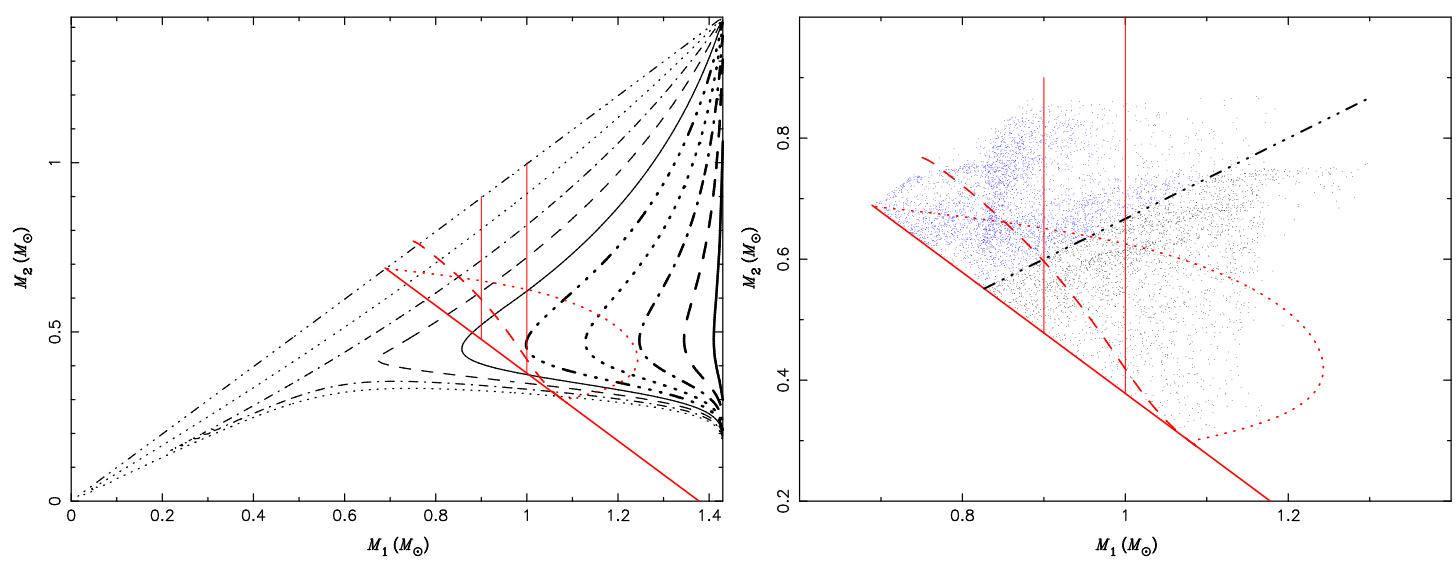

Fig. 1. - Left panel: constraints on the progenitors of SNe Ia of double CO WDs in the $M_{1}-M_{2}$ plane. The black lines are contours of the ejected mass fraction $1-\beta$ with values of, from left to right, $0.0,0.1, \ldots$ and 0.9 , respectively. The red lines mark contours of merger mass $M_{1}+M_{2}=M_{\mathrm{ch}}$ (conservative case, solid line), $M_{1}+\beta M_{2}=M_{\mathrm{ch}}$ (non-conservative, dotted line) and $M_{1}+\int \beta(t) \dot{M}_{2}(t) d t=M_{\mathrm{ch}}$ (non-conservative, dashed line). The vertical lines give the boundaries for $M_{1}=0.9$ and $1.0 M_{\odot}$. The areas to the right of each colour line represent $M \geq M_{\mathrm{ch}}$ and hence DD SN Ia progenitor candidates under the corresponding constraints. Right panel: CO DDs from our population synthesis with $M_{1}+M_{2}>M_{\mathrm{ch}}$ which can merge within a Hubble time, the blue dots have mass ratios $q=M_{2} / M_{1} \geq 2 / 3$. The black dash-dot-dot-dotted line shows the boundary of $q=2 / 3$, and the red lines are the same as those in the left-hand panel. 
orbital periods. Hence the $q>2 / 3$ constraint removes both the longest- and shortest-period binaries from the RLOF $+\mathrm{CE}$ channel, leaving a relatively larger number of progenitors with orbital periods of $\sim 3 \mathrm{hrs}$ upon emerging from the $\mathrm{CE}+\mathrm{CE}$ channel (corresponding delay times of $\log t=8.7)$. The 'dip' arises between the surviving $\mathrm{CE}+\mathrm{CE}$ and $\mathrm{RLOF}+\mathrm{CE}$ samples since short-orbital-period systems from the RLOF+CE channel have been removed.

\section{Conclusion}

We have shown that the DD model for SNe Ia is less effective than previously assumed if we consider the non-conservative nature of the DD merger process and constraints on triggering SN Ia explosions provided by recent SPH simulations. The overall SN Ia rate is reduced to a value $\approx 0.56-0.72$ that of previous studies (or $0.018-0.024 \mathrm{SNuM}$ ) by introducing the non-conservative approximation, while it is likely below 0.18 of that value (or $<0.006 \mathrm{SNuM}$ ) if we also consider the SPH constraints.

Theoretical estimates of the SN Ia rate from the SD model show a wide range of values from $\approx 0.001$ SNuM (Ruiter, Belczynski \& Fryer 2009) to > 0.01 SNuM ( Han \& Podsiadlowski 2004) (see also Mennekens et al. 2010), which strongly depends on the model assumptions and input parameters used in various studies. Wang et al. (2010) performed a study using the same synthesis model and Galactic (constant) star formation rate as used in this study, and found a rate of $0.029 \mathrm{SNuM}\left(2.15 \times 10^{-3} \mathrm{yr}^{-1}\right)$. By direct comparison, the DD model appears unlikely to be better than the SD model in explaining the SN Ia birthrate.

It was found by Pakmor et al. (2012) that an extended envelope enshrouding the merging WDs is not produced in violent merging events. However, we consider the possibility that a hot extended envelope may arise following a non-violent non-conservative DD merger. Since the disrupted material of the secondary must also contain most of the original orbital angular momentum, the outermost layers of the merger probably form a centrifugally supported disc. Our study therefore suggests that the lost or unaccreted material from the DD merger would take the form of a hot envelope plus disk, similar to that indicated by the SPH simulations of Yoon. Podsiadlowski \& Rosswog (2007) in which the scale of the hot envelope is small (i.e. $\leq 10^{10} \mathrm{~cm}$ ). The absence of early ultraviolet-optical emission in SN 2011fe (Nugent et al. 2011) is compatible with the DD model with a small extended envelope, while a disk would cause some continuum polarization such as that detected at red wavelengths in SN 2011fe (Smith et al. 2011), although other causes of polarization cannot be ruled out.

We thank the referee for his/her suggestions. This work is partly supported by the NSFC (Nos. 10973036, 11173055, 11033008 and 11003003), the CAS (No. KJCX2-YW-T24 
and the Talent Project of Western Light ), and the Project of Science and Technology from the Ministry of Education (211102).

\section{REFERENCES}

Chen, X., Han, Z., 2002, MNRAS,335, 948

Chen, X., Han, Z., 2003, MNRAS, 341, 662

Eggleton, P. P., Tout, C. A., Fitechett, M. J., 1989, ApJ, 347, 998

Geier, S., Heber, U., Kupfer, T., Napiwotzki, R., 2010, A\&A, 515, A37

Han, Z., 1998, MNRAS, 296, 1019

Han, Z., Podsiadlowski, Ph., 2004, MNRAS, 350, 1301

Han, Z., Tout, C. A., Eggleton, P. P., 2000, MNRAS, 319, 215

Han, Z., Webbink, R. F., 1999, A\&A, 349, L17 (HW)

Hurley, J. R., Tout, C. A., Pols, O. R., 2000, MNRAS, 315, 543

Hurley, J. R., Tout, C. A., Pols, O. R., 2002, MNRAS, 329, 897

Iben,I. Jr., Tutukov, A. V., 1984, ApJS, 54, 335

Kerzendorf, W.E., Yong, D., Schmidt, B.P., Jeffery, C.S., Anderson, J.P., Podsiadlowski, P., Gal-Yam, A., Simon, J.D., Silverman, J.M., Filippenko, A.V., Nomoto, K., Murphy, S.J., Bessell, M.S., Venn, K.A., Fioley, R.J., 2012, ApJ, submitted

Landau, L. D., Lifshitz, E. M., Classical Theory of Fields, Oxford: Pergamon Press, 1971

Livio, M., Soker, N., 1988, ApJ, 329, 764

Maoz, D., Mannucci, F., Li, W. et al, 2011, MNRAS, 412, 1508

Meng, X., Chen, X., Han, Z., 2009, MNRAS, 395, 2103

Mennekens, N., Vanbeveren, D., De Greve, J. P., De Donder E., 2010, A\&A, 515, A89

Miller, G. E., Scalo, J. M., 1979, ApJS, 41, 513

Napiwotzki, R., Karl, C. A., Nelemans, G., et al., 2007, ASPC, 372, 387 
Nelemans, G., Yungelson, L. R., Portegies Zwart, S. F., Verbunt, F., 2001, A\&A, 365, 491

Nomoto, K., Iben, I. Jr., 1985, ApJ, 297, 531

Nugent, P. E., Sullivan, M., Cenko, S. B. et al., 2011, Nature, 344, 480

Pakmor, R., Kromer, M., Röpke, F. K., et al., 2010, Nature, 463, 61

Pakmor, R., Hachinger, S., Röpke, F. K., Hillebrandt, W., 2011, A\&A, 528, A117

Pakmor, R., Kromer, M., Taubenberger, S., Sim, S. A., Röpke, F. K., Hillebrandt, W., 2012, ApJ, 747, L10

Piersanti, L., Gagliardi, S., Iben, I. J., Tornambé, A., 2003, ApJ, 598, 1229

Pols, O. R., Schröder, K. P., Hurley, J. R., Tout, C. A., Eggleton, P. P., 1998, MNRAS, 298, 525

Rodríguez-Gil, P., Santander-García, M., Knigge, C., et al. 2010, MNRAS, 407, L21

Ruiter, A. J., Belczynski, K., Fryer, C. L., 2009, ApJ, 699, 2026

Saio, H., Nomoto, K., 1985, A\&A150, L21

Schaefer, B. E., Pagnotta, A. 2012, Nature, 481, 164

Shen, K. J., Bildsten, L., Kasen, D., Quataert, E., 2012, ApJ, 748 , 35

Smith, P. S., Williams, G. G., Smith N. et al., 2011, arXiv:1111.6621

Timmes, F. X., Woosley, S. E., Taam, R. E., 1994, ApJ, 420, 348

Tutukow, A. V., Yungelson, L. R., 1981, Nauchnye Informatsii 49, 3

Tovmassian, G., Yungelson, L., Rauch, T., et al., 2010, ApJ, 714, 178

van den Heuvel, E. P. J., 1976, IAUS, 73, 35

van Kerkwijk, M. H., Chang, P., Justham, S., 2010, ApJ, 722, L157

Wang, B., Meng, X., Chen, X., Han, Z., 2009a, MNRAS, 395, 847

Wang, B., Chen, X., Meng, X., Han, Z., 2009b, ApJ, 701, 1540

Wang, B., Han, Z., 2012, NewA Review, 2012, 56, 122

Wang B., Liu Z., Han Z. et al., 2010, Sci. China Ser. G, 53, 586 
Webbink, R. F., 1984, ApJ, 277, 355

Yoon, S. C., Podsiadlowski, Ph., Rosswog, S., 2007, MNRAS, 380, 933

Yu, S., Jeffery, C.S., 2010, A\&A, 521, A85

Yungelson, L. R., Livio, M., Tutukov, A. V., Saffer, R. A., 1994, ApJ, 420, 336 

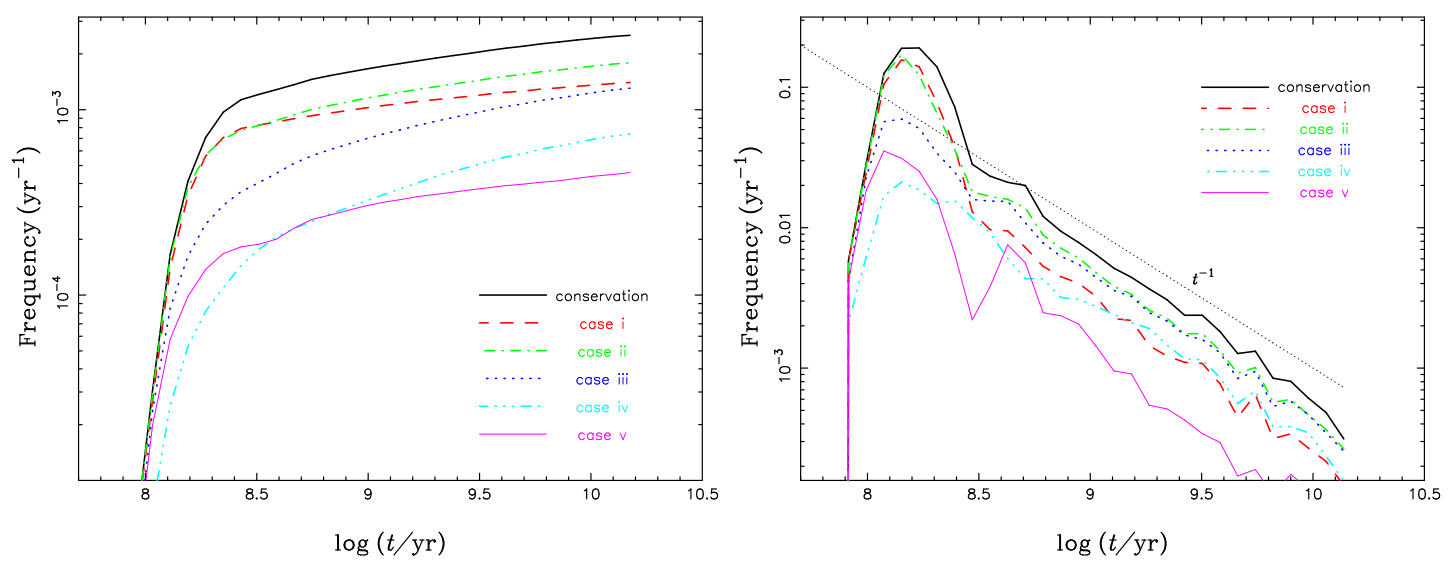

Fig. 2.- Left panel: the evolution of birthrate of SNe Ia for a constant Pop I star formation rate $\left(5 M_{\odot} \mathrm{yr}^{-1}\right)$ from the DD model. Right panel: as the left panel for a single starburst of $10^{11} M_{\odot}$. 